\title{
Mining the potential therapeutic targets for coronary artery disease by bioinformatics analysis
}

\author{
WENDONG WANG, ZHIWEI XU, XIAOBO ZHU and XIAOTONG CHANG
}

Department of Biochemistry, College of Laboratory Medicine, Hebei North University, Zhangjiakou, Hebei 075000, P.R. China

Received February 9, 2018; Accepted September 17, 2018

DOI: $10.3892 / \mathrm{mmr} .2018 .9551$

\begin{abstract}
The present study aimed to mine therapeutic molecular targets that play an important part in the progression of coronary artery disease (CAD). The gene expression profile GSE28829 dataset and the microRNA (miRNA) expression profile GSE59421 dataset were downloaded from the Gene Expression Omnibus (GEO) database. The GEO2R online analytical tool was used to identify differentially expressed genes (DEGs) and miRNAs (DEMs). The target genes of DEMs were identified using the miRWalk 2.0 web-based tool and 2 miRNA-gene regulatory networks were constructed using Cytoscape software. Subsequently, enriched Gene Ontology (GO) terms of miRNA-target DEGs were obtained using the Database for Visualization, Annotation and Integrated Analysis, and locations of these genes in the chromosomes were determined by Map Viewer. In the present study, 350 DEGs and 66 DEMs were screened. A total of 3,588 target genes were identified from the DEMs, and 57 of these target genes and established DEGs were identified to overlap. GO terms associated with 5 processes, and 4 types of composition were identified to be enriched in the miRNA-target DEGs. Furthermore, 26 miRNA-gene regulatory pairs were obtained between the 57 target genes and DEMs. The 26 miRNA-target DEGs were unevenly distributed, and no genes were located on the sex chromosomes. As a result of the present study, potential therapeutic targets for CAD were identified through bioinformatics analysis.
\end{abstract}

\section{Introduction}

Coronary artery disease (CAD), which is the most common type of cardiovascular disease, is the most prevalent cause of mortality worldwide (1). Recently, the incidence of CAD

Correspondence to: Professor Xiaotong Chang, Department of Biochemistry, College of Laboratory Medicine, Hebei North University, 11 Zuanshi South Road, Zhangjiakou, Hebei 075000, P.R. China

E-mail: changxt1212@vip.sina.com

Key words: coronary artery disease, microRNA, gene ontology, chromosomal location was demonstrated to have increased in China (2). Typically, the underlying mechanism of CAD involves a section of the coronary artery inter wall developing atherosclerosis. There are a variety of risk factors associated with CAD, including hypertension, obesity, smoking, family history of the disease (3), diabetes, lack of exercise, depression (4) and high blood lipids $(5,6)$. Although a number of advances in the understading of associated pathogeny, diagnosis and treatment of CAD have been described previously, current knowledge of the molecular mechanisms underlying CAD is insufficient for the development of improved treatment and diagnostic strategies for patients with CAD, and thus requires further investigation. Identification of the potential therapeutic targets for CAD remains clinically important.

DEGs have important roles in complex human diseases. MicroRNA (miRNAs) are a class of small noncoding RNA molecules that serve a crucial role in RNA silencing and the post-transcriptional regulation of gene expression by binding to the 3'-untranslated region of their target genes $(7,8)$. The identification of DEGs and DEMs associated with CAD may help to elucidate the underlying molecular mechanisms as well as discover novel biomarkers and therapies.

Recently, the present study performed a comprehensive analysis of global mRNA and miRNA expression profile datasets, including data from normal individuals and patients with CAD. A total of 350 mRNAs and 66 miRNAs were dysregulated in patients with CAD. Using these data, 2 networks of miRNA-gene associations were established. Through the identification of 26 miRNA-target DEGs, potential underlying molecular mechanisms and therapeutic targets for CAD were investigated.

\section{Materials and methods}

mRNA and miRNA expression datasets. In the present study, the GSE28829 mRNA (9) and GSE59421 miRNA (10) expression datasets were downloaded from the Gene Expression Omnibus (GEO) database (www.ncbi.nlm.nih.gov/geo). In the GSE28829 dataset, 16 atherosclerotic plaque samples from patients and 13 control samples were included and analyzed using the Affymetrix Human Genome U133 Plus 2.0 Array. In the GSE59421 dataset, the expression levels in platelets were identified in 33 male patients with premature CAD and 37 age- and sex-matched healthy controls, and were quantified using the agilent-021827 Human miRNA Microarray 
(V3) (miRBase release 12.0 miRNA ID version). These 2 datasets were indexed and loaded into Entrez GEO Profiles and Entrez GEO DataSets, which allows users to query and analyze the data using simple Boolean queries, and provides weblinks to free public raw data, accurate information, reliable data platforms and other National Center for Biotechnology Information resources wherever possible.

Microarray data mining. Analysis of differentially expressed genes (DEGs) was conducted for the mRNA and miRNA microarray data using GEO2R Online Analytical tool (https:/www.ncbi.nlm.nih.gov/geo/geo2r/). DEGs and differentially expressed miRNAs (DEMs) were identified using an adjusted $\mathrm{P}$-value $<0.05$ and a llog fold change (FC) $\mid>1$ as criteria.

Establishment of the miRNA-gene regulatory network. miRWalk2.0 (http://zmf.umm.uni-heidelberg.de/apps/zmf/ mirwalk2/) is a web-based tool developed by Dweep et al $(11,12)$ that supplies the largest available collection of predicted and experimentally verified miRNA-target interactions with various novel and unique features. In the present study, target genes of the DEMs were identified using miRWalk2.0. Furthermore, Cytoscape version 3.5.1 software (13) was used to establish the miRNA-gene regulatory network.

miRNA-target DEG protein-protein interactions. Search Tool for the Retrieval of Interacting Genes/Proteins (STRING; https://string-db.org/) is a database of known and predicted protein-protein interactions (14). In the present study, the protein-protein interactions were screened using STRING.

Functional enrichment analysis and Kyoto Encyclopedia of Genes and Genomes (KEGG) pathway analysis. Based on the Database for Visualization, Annotation and Integrated Analysis (DAVID; david.abcc.ncifcrf.gov/.) (15), the enriched Gene Ontology (GO) terms for miRNA-target DEGs were identified (only the GO terms that were enriched in $\geq 5$ genes were included). GO analysis is a commonly used approach for functional studies of large-scale transcriptomic data (16). The KEGG pathway database (17) contains information on networks of molecules or genes.

miRNA-target DEGs chromosomal location. The Map Viewer (https://www.ncbi.nlm.nih.gov/projects/mapview/) provides a wide variety of genome mapping and sequencing data (18). The locations of the miRNA-target DEGs on the chromosomes were identified using this resource.

\section{Results}

A total of 350 DEGs and 66 DEMs are identified. The available numerical mRNA and miRNA expression values were used to identify DEGs and DEMs. A total of 59 upregulated and 291 downregulated DEGs, and 30 upregulated and 36 downregulated DEMs were identified in patients with CAD compared with the normal controls. The top 10 DEGs and DEMs are presented in Table I $(\mathrm{P}<0.05 ; \mid \log \mathrm{FCl}>1)$.

miRNA-gene regulation network and protein interactions of 57 overlapping DEGs. Using miRWalk2.0, 3,588 target genes of the DEMs were identified, and 57 of these target genes were demonstrated to overlap with the identified DEGs. In the group of 57 overlapping DEGs, a protein-protein interaction network of 26 DEGs was established using STRING. Furthermore, 26 miRNA-gene pairs were obtained among the 26 aforementioned DEGs and 19 DEMs. The 19 DEMs were demonstrated to regulate multiple genes, thereby forming a considerable network (Fig. 1).

GO and KEGG pathway analysis. Using DAVID, 40 enriched GO process terms were obtained for miRNA-targeting DEGs. The five most significantly enriched GO terms, including 'regulation of cell communication', 'regulation of signaling', 'regulation of response to stimulus', 'positive regulation of biological process' and 'positive regulation of cellular process', are presented in Fig. 2A. A total of 4 enriched GO component terms were obtained for miRNA-target DEGs, including 'low-density lipoprotein particle', 'plasma membrane', 'cell periphery' and 'membrane' (Fig. 2B). KEGG pathway analysis identified 3 genes [hexokinase 2 (HK2), phosphatidylinositol-4,5-biphosphate 3-kinase catalytic subunit $\gamma$ (PIK3CG), and suppressor of cytokine signaling 3 (SOCS3)] that were present in Type II diabetes mellitus network and 3 other genes, ATPase $\mathrm{Na}+\mathrm{K}+$ transporting subunit $\alpha 2, \mathrm{HK} 2$ and PIK3CG that were present in the carbohydrate digestion and absorption network.

Furthermore, the miRNA-gene regulatory network of the 26 miRNA-gene pairs was established using Cytoscape version 3.5.1 software (Fig. 3). The 26 regulatory pairs between DEMs and their target genes were identified, including hsa-miR-199a-5p-apolioprotein E (APOE), hsa-miR-106a-branched chain amino acid transaminase 1 (BCAT1), hsa-miR-1234-cytohesin 1 interacting protein (CYTIP), hsa-miR-1249-macrophage scavenger receptor 1 (MSR1), hsa-miR-142-3p-ecotropic viral integration site 2B (EVI2B), hsa-miR-142-3p-myristoylated alanine rich protein kinase C substrate (MARCKS), hsa-miR-142-3p-Phosphatidylinositol-4,5-bisphosphate 3-kinase catalytic subunit gamma isoform (PIK3CG), hsa-miR-152-MAF basic leucine zipper (BZIP) transcription factor B (MAFB), hsa-miR-215-DEP domain containing MTOR interacting protein (DEPTOR), hsa-miR-215-HOP homeobox (HOPX), hsa-miR-22-colony stimulating factor 1 receptor (CSF1R), hsa-miR-221-coronin $1 \mathrm{~A}$ (CORO1A), hsa-miR-221-Suppressor of cytokine signaling 3 (SOCS3), hsa-miR-221-T cell lymphoma invasion and metastasis 1 (TIAM1), hsa-miR-30b-immunoglobulin superfamily member 6 (IGSF6), hsa-miR-30c-C-X-C motif chemokine ligand 16 (CXCL16), hsa-miR-30e-Serpin family E member 2 (SERPINE2), hsa-miR-323a-3p-phospholipase A2 group VII (PLA2G7), hsa-miR-329-ecotropic viral integration site 2A (EVI2A), hsa-miR-33a-chromosome 15 open reading frame 48 (C15orf48), hsa-miR-33a-FYN binding protein 1 (FYB), hsa-miR-376-death associated protein kinase 1 (DAPK1), hsa-miR-484-hematopoietic cell-specific Lyn substrate 1 (HCLS1), hsa-miR-484-HK2, hsa-miR-486-3p-netrin 1 (NTN1) and hsa-miR-616-lysozyme (LYZ).

miRNA-target DEG chromosomal location. The 26 DEGs in the miRNA-gene pairs were located on the chromosomes by Map Viewer (Fig. 4). The chromosomal locations of these genes 
Table I. Top 10 most differentially expressed genes and differentially expressed miRNAs.

\begin{tabular}{|c|c|c|c|c|c|}
\hline Gene & P-value & $\log \mathrm{FC}$ & miRNA & P-value & $\log \mathrm{FC}$ \\
\hline $\mathrm{C} 2$ & $1.6 \times 10^{-10}$ & -1.25626406 & hsa-miR-221 & $1.13 \times 10^{-4}$ & -1.2728544 \\
\hline DENND2D & $5.16 \times 10^{-10}$ & -1.15465237 & hsa-miR-1246 & $2.52 \times 10^{-4}$ & -1.6266646 \\
\hline ADAP2 & $2.45 \times 10^{-9}$ & -1.22371715 & hsa-miR-425 & $8.73 \times 10^{-4}$ & -1.1111774 \\
\hline JAK3 & $3.09 \times 10^{-9}$ & -1.09039561 & hsa-miR-505 & $1.54 \times 10^{-3}$ & -1.1639686 \\
\hline SLAMF8 & $4.26 \times 10^{-9}$ & -2.07254568 & hsa-miR-598 & $1.93 \times 10^{-3}$ & -1.2433251 \\
\hline CD68 & $4.51 \times 10^{-9}$ & -1.13556898 & hsa-miR-431* & $3.26 \times 10^{-3}$ & 1.0564151 \\
\hline LINC01094 & $6.25 \times 10^{-9}$ & -1.10497076 & hsa-miR-25 & $3.85 \times 10^{-3}$ & -1.0901694 \\
\hline SERPINA1 & $8.24 \times 10^{-9}$ & -1.73250228 & hsa-miR-30e & $4.66 \times 10^{-3}$ & -1.1414787 \\
\hline VAMP8 & $8.69 \times 10^{-9}$ & -1.82538528 & hsa-miR-370 & $5.2 \times 10^{-3}$ & 1.2500556 \\
\hline C3AR1 & $1.14 \times 10^{-8}$ & -2.22628842 & hsa-miR-1274a & $5.36 \times 10^{-3}$ & -1.27226 \\
\hline
\end{tabular}

FC, fold-change; hsa, homo sapiens; miRNA, microRNA.

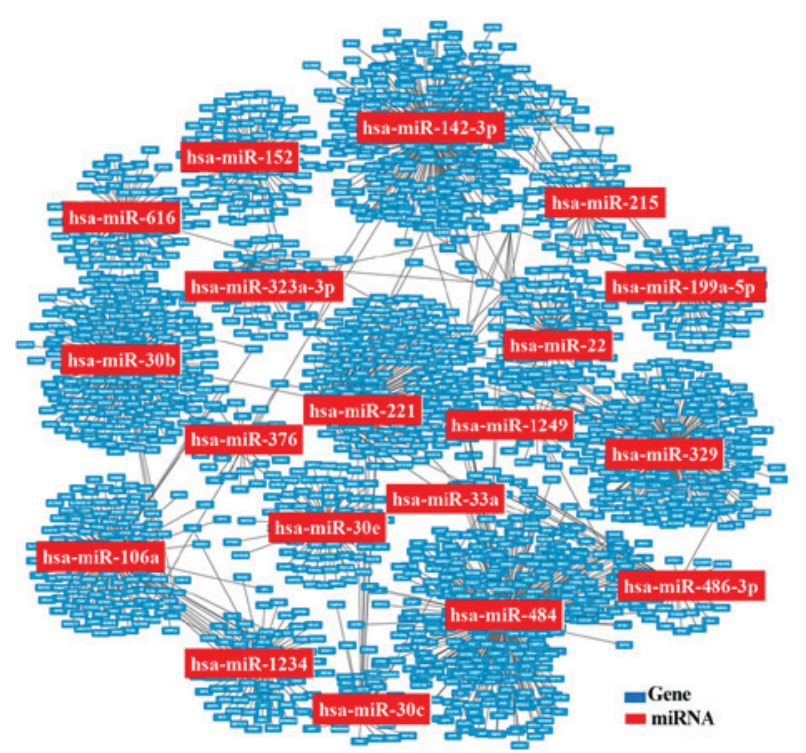

Figure 1. DEMs regulate multiple genes. Red squares represent DEMs and blue squares denote DEGs. Each line (edge) is a pairwise connection between 2 genes, or between DEMs and DEGs. The figure was plotted using Cytoscape version 3.5.1 software. hsa, homo sapiens; miR, microRNA; DEMs, differentially expressed miRNAs; DEGS, differentially expressed genes.

were inhomogeneous, and they were primarily distributed on chromosomes $17,2,5,6,12,16,1,3,4,7,8,9,15,19,20$, and 21 . None of the associated genes were distributed on sex chromosomes.

\section{Discussion}

miRNAs are crucial and evolutionarily conserved components of gene regulation (19). Up- or downregulated expression levels of specific miRNAs were observed in diseased human hearts, implying their involvement in cardiomyopathies (20). $\mathrm{CAD}$ is an ischemic heart disease that occurs when part of the arterial internal wall develops atherosclerosis, which is associated with lipid retention and inflammation. Murine miRNA-712 is a potential biomarker for atherosclerosis (21). However, the detailed mechanism underlying the mechanism by which miRNAs regulate the expression of CAD-associated genes and gene products requires thorough investigation.

In the present study, a total of 59 upregulated and 291 downregulated DEGs, and 30 upregulated and 36 downregulated DEMs were identified in patients with CAD compared with normal controls. The GO analysis indicated that several significantly enriched terms from the categories of regulation of cell communication, regulation of signaling, regulation of response to stimulus, positive regulation of biological process, and positive regulation of cellular process were markedly over-represented in patients with CAD, suggesting that these biological processes are important topics for future study.

The present study also identified the interactions and associations among proteins, which were encoded by 26 DEGs in the miRNA-gene pairs. In addition, the 19 miRNAs (which target the 26 DEGs) also regulate a large quantity of genes.

Apolipoprotein E is encoded by the APOE gene, and serves as a shuttle lipid in the bloodstream (22). Maintaining constant levels of cholesterol is very important for avoiding cardiovascular disease. Carriers of the APOEe4 allele exhibit increased risks of developing atherosclerosis; an abnormal expression of APOE is associated with fatty deposition, which increases the risk of heart attack and stroke (23). In addition, previous studies have identified that mutated APOE causes an increased risk of Alzheimer's disease in individuals; however, the mechanism is unclear (24). Thus, it may be suggested that the APOE gene is associated with Alzheimer's disease and CAD. The BCAT1 gene encodes a form of transaminase to catalyze the branched-chain amino acid transamination, which when defective is prone to 2 types of disease; hypervalinemia and hyperleucine-isoleucinemia $(25,26)$. The coding products of the MSR1 gene are membrane glycoproteins, which are associated with atherosclerosis, Alzheimer's disease and host defense (27). DEPTOR has been considered as an endogenous regulator overexpressed in multiple myeloma cells (28). Increased levels of CSF1R were identified in microglia in Alzheimer's disease (29). A number of types of cytokines [interleukin (IL)6, IL10, interferon (IFN)- $\gamma$ ] may induce the expression of SOCS3 gene. Knocking out 
A

A Intracellular signal transduction

$$
\begin{array}{r}
\text { Cell adhesion } \\
\text { Innate immune response }
\end{array}
$$

Regulation of multicellular organismal development

Regulation of cellular component size

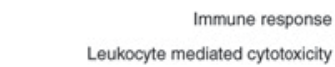

Negative regulation of signal transduction

Positive regulation of cellular process

Regulation of signal transduction

Cellular response to organic substance

Positve regulation of cell differentiation

Negative regulation of cell communication

Regulation of cell migration

Regulation of ion transport

Regulation of cellular component movement

Positive regulation of developmental process

Regulation of homeostatic process

Regulation of cell communication
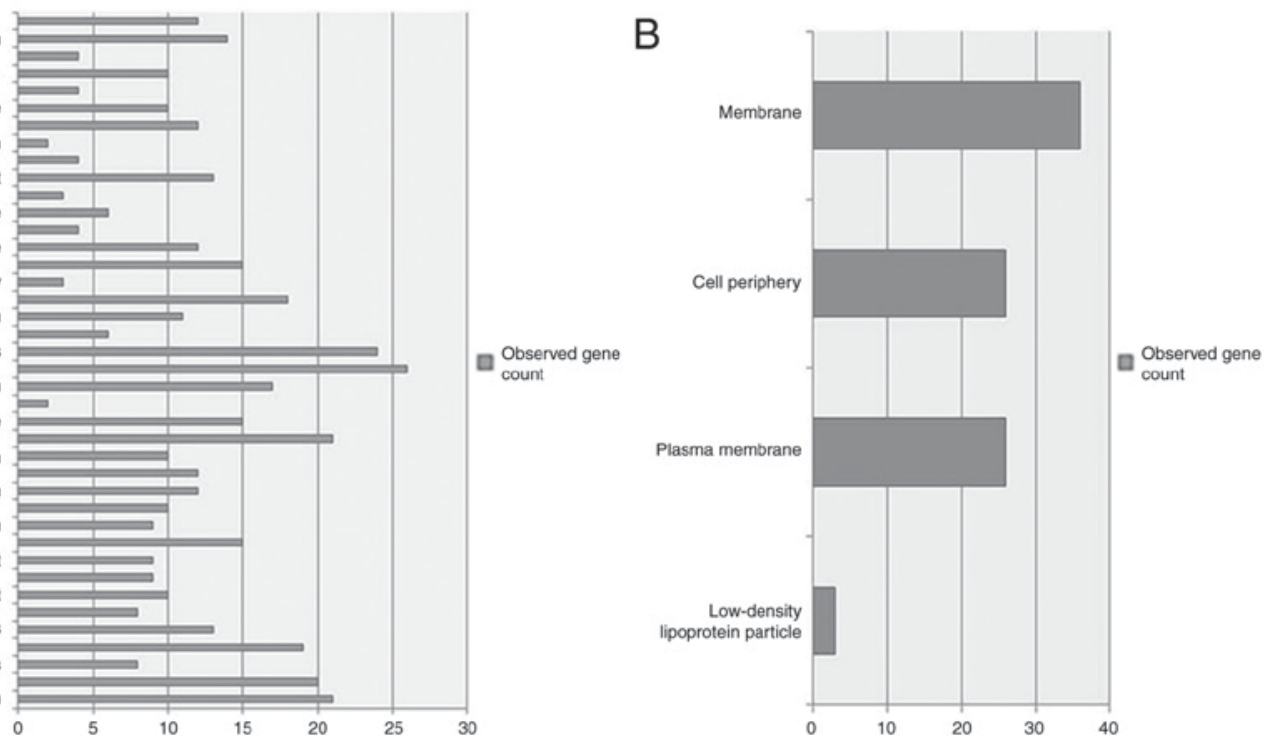

Figure 2. Enriched GO process terms and component terms of miRNA-target differentially expressed genes. (A) Enriched GO process terms and (B) enriched GO component terms of miRNA-target differentially expressed genes obtained from the Database for Visualization, Annotation and Integrated Analysis. GO, gene ontology; miRNA, microRNA.

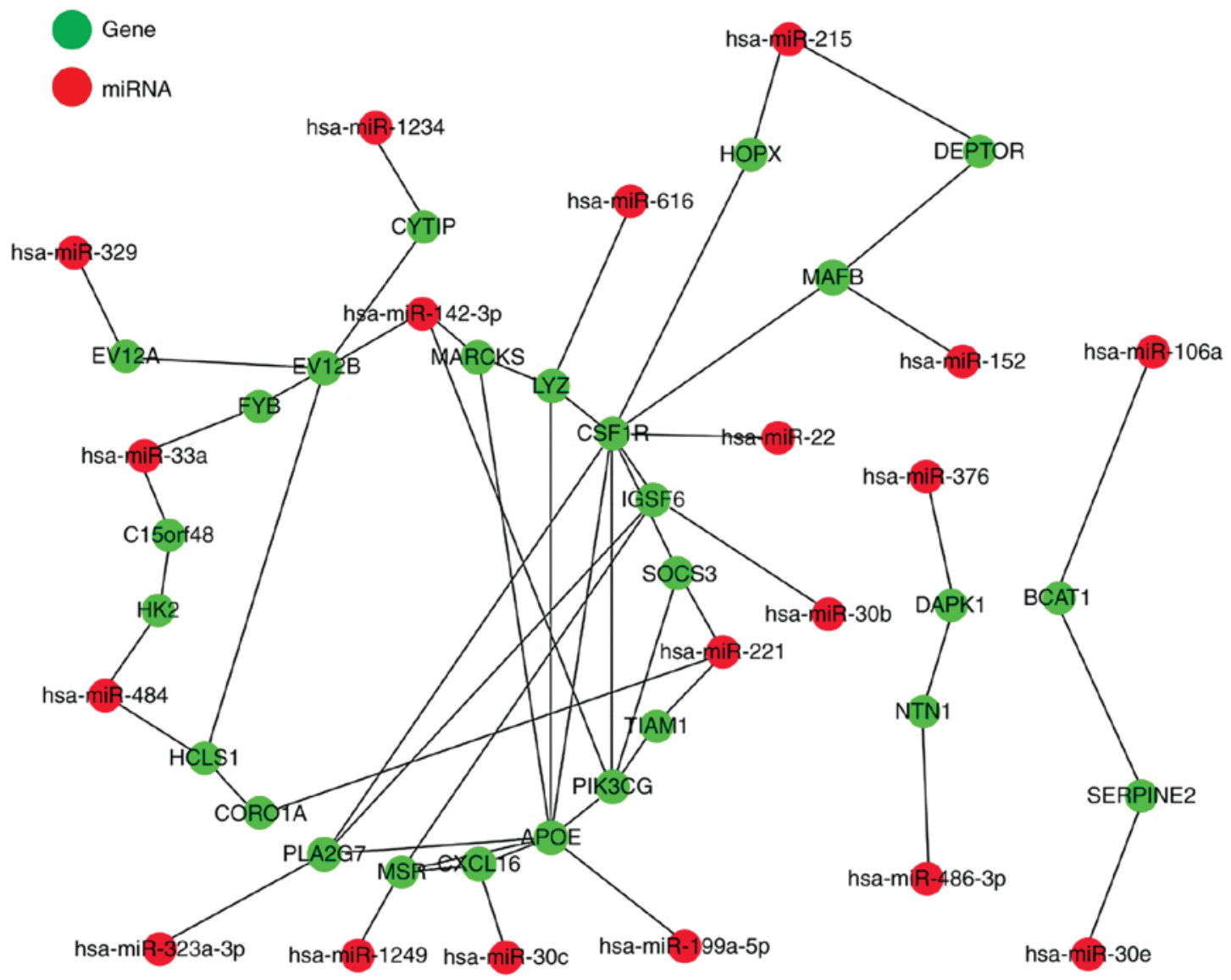

Figure 3. miRNA-gene regulatory network of 26 miRNA-gene pairs. Green circles represent genes and red circles denote miRNA. Each line (edge) is a pairwise connection between two genes, or between differentially expressed miRNAs and differentially expressed genes. The figure was plotted using Cytoscape version 3.5.1 software. hsa, homo sapiens; miR, microRNA.

the SOCS3 gene prevents insulin resistance in obesity (30). The IGSF6 gene is associated with inflammatory bowel disease (31). HK2 is the first and rate-limiting enzyme in the Embden-Meyerhof-Parnas pathway (32). DAPK1 is a positive mediator of $\gamma$-interferon-induced programmed cell death. Diseases identified to be associated with HCLS1 include spherocytosis, type 1 and congenital hemolytic anemia (33). The product of the HCLS1 gene serve a role in antigen receptor 

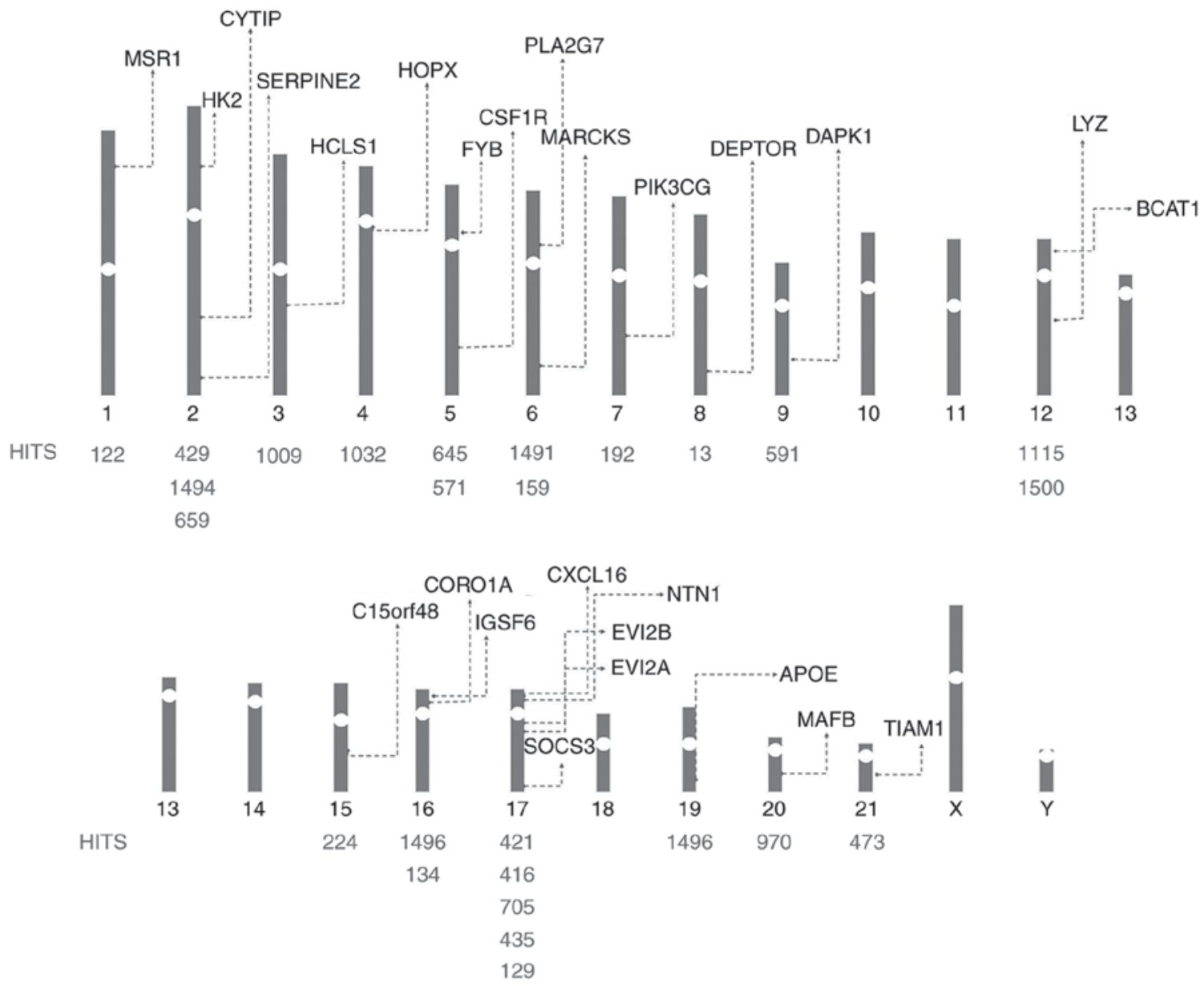

Figure 4. microRNA-target differentially expressed genes chromosomal location. Black rectangles represent chromosomes and white spots represents centromeres. The arrows signify the specific locations of the genes.

signaling for clonal expansion and deletion in lymphoid cells (34). Platelet-activating factor acetylhydrolase deficiency may be caused by the defective PLA2G7 gene, in which the associated pathways are the phospholipase- $\mathrm{C}$ pathway and sweet taste receptor signaling (35). Oxidation of low-density lipoproteins is an initial step of atherogenesis that generates pro-inflammatory phospholipids, including platelet-activating factor and its analogs (36). GO annotations associated with EVI2A include transmembrane signaling receptor activity (37). Mutations in SERPINE2 may cause a range of diseases; the $\alpha$-antitrypsin deficiency is one of the most common hereditary diseases associated with this gene (38). CXCL16 expression is induced by the inflammatory cytokines IFN- $\gamma$ and tumor necrosis factor- $\alpha$ (39). In patients with systemic lupus erythematosus, the differential expression of the FYB gene encodes a protein that participates in the T-cell signaling cascade and in IL-2A expression modulation (40). The $\mathrm{C} 15$ orf 48 gene was first identified in a study of human esophageal squamous cell carcinoma tissues (41). The HOPX gene is involved in the malignant conversion of placental trophoblasts (42). The protein encoded by CYTIP is weakly expressed in resting natural killer and $\mathrm{T}$ cells, which contain 2 leucine zipper domains and a putative C-terminal nuclear targeting signal (43). CYTIP is associated with amplification and expansion of oncogenic pathways, and exhibits metastatic traits (43). The gene product of the EVI2B gene has been studied extensively regarding its role in neurofibromatosis, leukemia, and myeloid leukemia (44). The MARCKS gene product serves important roles in cell shape, cell motility, secretion, transmembrane transport, regulation of cell cycle and neural development (45). The PIK3CG gene product is an enzyme that phosphorylates phosphoinositides on the 3-hydroxyl group of the inositol ring, PIK3CG SNPs (rs1129293 and rs17398575) increase the risk of ischemia in patients suffering from coronary heart disease (44). MAFB is a bZIP transcription factor that serves an important role in the regulation of lineage-specific hematopoiesis, and rs2902940A alleles of the MAFB gene result in decreased serum ApoAI levels in controls and an increased risk of CAD (46). The encoded nuclear protein represses ETS1-mediated transcription of erythroid-specific genes in myeloid cells (47). CORO1A has been implicated in T-cell mediated immunity and mitochondrial apoptosis (48). TIAM1 has been identified as a specific activator of the Rho-like GTPase Rac and is implicated in the regulation of different cell biological functions, including cell polarity, adhesion, migration, invasion, metastasis, and carcinogenesis (49). NTN1 is included in a family of laminin-associated secreted proteins (50). The LYZ protein exhibits antibacterial activity against certain bacterial species. Missense mutations in the LYZ gene have been identified in heritable renal amyloidosis. miRNAs serve as stimulating factors at various stages of atherosclerosis to regulate a number of genes, thereby regulating endothelial cells, vascular smooth muscle cells, and 
the function of macrophages, which control the occurrence of atherosclerosis (51).

The chromosomal locations of these aforementioned 26 genes are inhomogeneous, and they are primarily distributed on chromosomes $17,2,5,6,12,16,1,3,4,7,8$, 9, 15, 19, 20 and 21. No miRNA-target DEGs were identified on the sex chromosomes, which suggested that CAD may be independent of sex. in CAD. Finally, the results of the study may be a useful resource for understanding the functions of miRNA and potential therapeutic targets in the pathophysiology of CAD, but additional molecular analyses are required. The affected interactions of the 26 miRNA-gene pairs identified in the present study may be validated in vitro by dual-luciferase reporter gene assays, and the functions of the 26 miRNAs and their target genes will be confirmed in vivo and in vitro, so that potential therapeutic targets may be identified and effective targeting of the affected biological pathways may be achieved for the treatment and improved prognosis of CAD.

\section{Acknowledgements}

Not applicable.

\section{Funding}

The present study was supported by a grant from the Key Programs of the Educational Commission of Hebei Province of China (grant no. ZD2018076) and Youth Science and Technology Project of Health and Municipal Commission of Population and Family of Hebei Province of China (grant no. 20180811).

\section{Availability of data and materials}

The datasets generated and/or analyzed during the current study are available in the Gene Expression Omnibus repository [GSE28829 dataset, https://www.ncbi.nlm. nih.gov/geo/query/acc.cgi?acc=GSE28829; GSE59421 dataset, https://www.ncbi.nlm.nih.gov/geo/query/acc. cgi?acc=GSE59421].

\section{Authors' contributions}

WW performed the bioinformatics analyses. ZX mined and downloaded the data from the Gene Expression Omnibus database. XZ analyzed and interpreted the data. $\mathrm{XC}$ identified differentially expressed genes targeted by microRNAs associated with coronary heart disease and analyzed the results. WW was a major contributor in the writing of the manuscript. All authors read and approved the final manuscript.

\section{Ethics approval and consent to participate}

Not applicable.

\section{Patient consent for publication}

Not applicable.

\section{Competing interests}

The authors declare that they have no competing interests.

\section{References}

1. Mendis S, Puska P and Norrving B: Global: Atlas on cardiovascular disease prevention and control. Geneva: World Health Organization in collaboration with the World Heart Federation and the World Stroke Organization, pp3-18, 2011.

2. Ding D, Wang M, Su D, Hong C, Li X, Yang Y, Zhang Y, Hu G and Ling W: Body mass index, high-sensitivity C-reactive protein and mortality in Chinese with coronary artery disease. PLoS One 10: e0135713, 2015.

3. Centers for Disease Control and Prevention (CDC): Prevalence of coronary heart disease-United States, 2006-2010. MMWR Morb Mortal Wkly Rep 60: 1377-1381, 2011.

4. GBD 2015 Disease and Injury Incidence and Prevalence Collaborators: Global, regional, and national incidence, prevalence, and years lived with disability for 310 diseases and injuries, 1990-2015: A systematic analysis for the global burden of disease study 2015. Lancet 388: 1545-1602, 2016.

5. Kontos MC, Diercks DB and Kirk JD: Emergency department and office-based evaluation of patients with chest pain. Mayo Clin Proc 85: 284-299, 2010.

6. Dai X, Wiernek S, Evans JP and Runge MS: Genetics of coronary artery disease and myocardial infarction. World J Cardiol 8: $1-23,2016$.

7. Zimmerman $\mathrm{AL}$ and Wu S: MicroRNAs, cancer and cancer stem cells. Cancer Lett 300: 10-19, 2011.

8. Esteller M: Non-coding RNAs in human disease. Nat Rev Genet 12: 861-874, 2011.

9. Doring Y, Manthey HD, Drechsler M, Lievens D, Megens RT, Soehnlein O, Busch M, Manca M, Koenen RR, Pelisek J, et al: Auto-antigenic protein-DNA complexes stimulate plasmacytoid dendritic cells to promote atherosclerosis. Circulation 125: 1673-1683, 2012.

10. Kok MG, Halliani A, Moerland PD, Meijers JC, Creemers EE and Pinto-Sietsma SJ: Normalization panels for the reliable quantification of circulating microRNAs by RT-qPCR. FASEB J 29: 3853-3862, 2015.

11. Dweep H, Sticht C, Pandey P and Gretz N: miRWalk-database: Prediction of possible miRNA binding sites by 'walking' the genes of 3 genomes. J Biomed Inform 44: 839-837, 2011.

12. Dweep H and Gretz N: miRWalk2.0: A comprehensive atlas of microRNA-target interactions. Nat Methods 12: 697-697, 2015.

13. Shannon P, Markiel A, Ozier O, Baliga NS, Wang JT, Ramage D, Amin N, Schwikowski B and Ideker T: Cytoscape: A software environment for integrated models of biomolecular interaction networks. Genome Res 13: 2498-2504, 2003.

14. Szklarczyk D, Morris JH, Cook H, Kuhn M, Wyder S, Simonovic M, Santos A, Doncheva NT, Roth A, Bork P, et al: The STRING database in 2017: Quality-controlled protein-protein association networks, made broadly accessible. Nucleic Acids Res 45: D362-D368, 2017.

15. Huang da W, Sherman BT and Lempicki RA: Systematic and integrative analysis of large gene lists using DAVID bioinformatics resources. Nat Protoc 4: 44-57, 2009.

16. Hulsegge I, Kommadath A and Smits MA: Globaltest and GOEAST: Two different approaches for Gene Ontology analysis. BMC Proc 3 (Suppl 4): S10, 2009.

17. Ogata H, Goto S, Sato K, Fujibuchi W, Bono H and Kanehisa M: KEGG: Kyoto encyclopedia of genes and genomes. Nucleic Acids Res 27: 29-34, 1999.

18. Wolfsberg TG: Using the NCBI Map Viewer to browse genomic sequence data. Curr Protoc Hum Genet Chapter 18: Unit 18.5, 2011.

19. Peterson KJ, Dietrich MR and McPeek MA: MicroRNAs and metazoan macroevolution: Insights into canalization, complexity, and the Cambrian explosion. Bioessays 31: 736-747, 2009.

20. Tatsuguchi M, Seok HY, Callis TE, Thomson JM, Chen JF, Newman M, Rojas M, Hammond SM and Wang DZ: Expression of microRNAs is dynamically regulated during cardiomyocyte hypertrophy. J Mol Cell Cardiol 42: 1137-1141, 2007.

21. Insull W Jr: The pathology of atherosclerosis: Plaque development and plaque responses to medical treatment. Am J Med 122 (1 Suppl): S3-S14, 2009. 
22. Phillips MC: Apolipoprotein E isoforms and lipoprotein metabolism. IUBMB Life 66: 616-623, 2014.

23. Eichner JE, Dunn ST, Perveen G, Thompson DM, Stewart KE and Stroehla BC: Apolipoprotein E polymorphism and cardiovascular disease: A HuGE review. Am J Epidemiol 155: 487-495, 2002.

24. Mahley RW, Weisgraber KH and Huang Y: Apolipoprotein E4: A causative factor and therapeutic target in neuropathology, including Alzheimer's disease. Proc Natl Acad Sci USA 103: 5644-5651, 2006.

25. Chang IW, Wu WJ, Wang YH, Wu TF, Liang PI, He HL, Yeh BW and Li CF: BCAT1 overexpression is an indicator of poor prognosis in patients with urothelial carcinomas of the upper urinary tract and urinary bladder. Histopathology 68: 520-532, 2016.

26. Goto M, Miyahara I, Hirotsu K, Conway M, Yennawar N, Islam MM and Hutson SM: Structural determinants for branched-chain aminotransferase isozyme-specific inhibition by the anticonvulsant drug gabapentin. J Biol Chem 280 : 37246-37256, 2005

27. Ashkenas J, Penman M, Vasile E, Acton S, Freeman M and Krieger M: Structures and high and low affinity ligand binding properties of murine type I and type II macrophage scavenger receptors. J Lipid Res 34: 983-1000, 1993.

28. Peterson TR, Laplante M, Thoreen CC, Sancak Y, Kang SA, Kuehl WM, Gray NS and Sabatini DM: DEPTOR is an mTOR inhibitor frequently overexpressed in multiple myeloma cells and required for their survival. Cell 137: 873-886, 2009.

29. Mitrasinovic OM, Grattan A, Robinson CC, Lapustea NB Poon C, Ryan H, Phong C and Murphy GM Jr: Microglia overexpressing the macrophage colony-stimulating factor receptor are neuroprotective in a microglial-hippocampal organotypic coculture system. J Neurosci 25: 4442-4451, 2005.

30. Jorgensen SB, O'Neill HM, Sylow L, Honeyman J, Hewitt KA, Palanivel R, Fullerton MD, Öberg L, Balendran A, Galic S, et al Deletion of skeletal muscle SOCS3 prevents insulin resistance in obesity. Diabetes 62: 56-64, 2013.

31. King K, Moody A, Fisher SA, Mirza MM, Cuthbert AP, Hampe J, Sutherland-Craggs A, Sanderson J, MacPherson AJ, Forbes A, et al: Genetic variation in the IGSF6 gene and lack of association with inflammatory bowel disease. Eur J Immunogenet 30: 187-190, 2003

32. Ahn KJ, Kim J, Yun M, Park JH and Lee JD: Enzymatic properties of the $\mathrm{N}$ - and $\mathrm{C}$-terminal halves of human hexokinase II. BMB Rep 42: 350-355, 2009.

33. Feinstein E, Druck T, Kastury K, Berissi H, Goodart SA, Overhauser J, Kimchi A and Huebner K: Assignment of DAP1 and DAPK-genes that positively mediate programmed cell death triggered by IFN-gamma-to chromosome regions $5 \mathrm{p} 12.2$ and 9q34.1, respectively. Genomics 29: 305-307, 1995.

34. van Rossum AG, Schuuring-Scholtes E, van Buuren-van Seggelen V, Kluin PM and Schuuring E: Comparative genome analysis of cortactin and HS1: The significance of the F-actin binding repeat domain. BMC Genomics 6: 15, 2005.

35. Mohler ER III, Ballantyne CM, Davidson MH, Hanefeld M, Ruilope LM, Johnson JL and Zalewski A; Darapladib Investigators: The effect of darapladib on plasma lipoprotein-associated phospholipase A2 activity and cardiovascular biomarkers in patients with stable coronary heart disease or coronary heart disease risk equivalent: The results of a multicenter, randomized, double-blind, placebo-controlled study. J Am Coll Cardiol 51: 1632-1641, 2008.

36. Ninio E, Tregouet D, Carrier JL, Stengel D, Bickel C, Perret C, Rupprecht HJ, Cambien F, Blankenberg S and Tiret L: Platelet-activating factor-acetylhydrolase and PAF-receptor gene haplotypes in relation to future cardiovascular event in patients with coronary artery disease. Hum Mol Genet 13: 1341-1351, 2004
37. Cawthon RM, O'Connell P, Buchberg AM, Viskochil D, Weiss RB, Culver M, Stevens J, Jenkins NA, Copeland NG and White R: Identification and characterization of transcripts from the neurofibromatosis 1 region: The sequence and genomic structure of EVI2 and mapping of other transcripts. Genomics 7: $555-565,1990$

38. Janciauskiene SM, Bals R, Koczulla R, Vogelmeier C, Köhnlein T and Welte T: The discovery of $\alpha 1$-antitrypsin and its role in health and disease. Respir Med 105: 1129-1139, 2011.

39. Abel S, Hundhausen C, Mentlein R, Schulte A, Berkhout TA, Broadway N, Hartmann D, Sedlacek R, Dietrich S, Muetze B, et al: The transmembrane CXC-chemokine ligand 16 is induced by IFN-gamma and TNF-alpha and shed by the activity of the disintegrin-like metalloproteinase ADAM10. J Immunol 172: 6362-6372, 2004.

40. Addobbati C, Brandão LA, Guimarães RL, Pancotto JA, Donadi EA, Crovella S, Segat L and Sandrin-Garcia P: FYB gene polymorphisms are associated with susceptibility for systemic lupus erythemathosus (SLE). Hum Immunol 74: 1009-1014, 2013.

41. Zimmer A, Bouley J, Le Mignon M, Pliquet E, Horiot S, Turfkruyer M, Baron-Bodo V, Horak F, Nony E, Louise A, et al: A regulatory dendritic cell signature correlates with the clinical efficacy of allergen-specific sublingual immunotherapy. J Allergy Clin Immunol 129: 1020-1030, 2012.

42. Asanoma K, Kato H, Inoue T, Matsuda T and Wake N: Analysis of a candidate gene associated with growth suppression of choriocarcinoma and differentiation of trophoblasts. J Reprod Med 49: 617-626, 2004.

43. Heufler C, Ortner D and Hofer S: Cybr, CYTIP or CASP: An attempt to pinpoint a molecule's functions and names. Immunobiology 213: 729-732, 2008

44. Zjablovskaja P, Kardosova M,DanekP, AngelisovaP, BenoukrafT, Wurm AA, Kalina T, Sian S, Balastik M, Delwel R, et al: EVI2B is a $\mathrm{C} / \mathrm{EBP} \alpha$ target gene required for granulocytic differentiation and functionality of hematopoietic progenitors. Cell Death Differ 24: 705-716, 2017.

45. Prieto D and Zolessi FR: Functional diversification of the four MARCKS family members in zebrafish neural development. J Exp Zool B Mol Dev Evol 328: 119-138, 2016.

46. Yang Q, Yin RX, Zhou YJ, Cao XL, Guo T and Chen WX: Association of polymorphisms in the MAFB gene and the risk of coronary artery disease and ischemic stroke: A case-control study. Lipids Health Dis 14: 79, 2015.

47. Dwyer J, Li H, Xu D and Liu JP: Transcriptional regulation of telomerase activity: Roles of the Ets transcription factor family. Ann N Y Acad Sci 1114: 36-47, 2007

48. Kerber RA, OBrien E and Cawthon RM: Gene expression profiles associated with aging and mortality in humans. Aging Cell 8: 239-250, 2009.

49. Xu Z, Gakhar L, Bain FE, Spies M and Fuentes EJ: The Tiam1 guanine nucleotide exchange factor is auto-inhibited by its pleckstrin homology coiled-coil extension domain. J Biol Chem 292: 17777-17793, 2017.

50. Ren XR, Hong Y, Feng Z, Yang HM, Mei L and Xiong WC: Tyrosine phosphorylation of netrin receptors in netrin-1 signaling. Neurosignals 16: 235-345, 2008.

51. Jean E, Ebbo M, Valleix S, Benarous L, Heyries L, Grados A, Bernit E, Grateau G, Papo T, Granel B, et al: A new family with hereditary lysozyme amyloidosis with gastritis and inflammatory bowel disease as prevailing symptoms. BMC Gastroenterol 14: 159,2014

This work is licensed under a Creative Commons Attribution-NonCommercial-NoDerivatives 4.0 International (CC BY-NC-ND 4.0) License. 